\title{
Efecto de la suplementación preparto con cloruro de calcio sobre la concentración sérica de minerales y aspectos productivos en vacas Carora
}

\author{
Effect of pre-partum supplementation with calcium chloride on the serum \\ concentration of minerals and productive aspects in Carora cows
}

Villany Villarreal ${ }^{1}$, Aura López-Ortega ${ }^{1}$, Adelys Márquez $^{1}$, Ysabel Márquez ${ }^{1}$

\section{Resumen}

El objetivo del estudio fue evaluar el efecto de la suplementación preparto con una dieta aniónica sobre concentraciones séricas de minerales durante el periparto, ocurrencia de hipocalcemia subclínica, retención de membranas fetales (RMF) y producción de leche en los primeros 100 días posparto. Se empleó un diseño completamente al azar, utilizando 24 vacas Carora distribuidas en dos grupos similares: un grupo suplementado con $100 \mathrm{~g}$ de cloruro de calcio/vaca/día entre los 21 días antes de la fecha probable de parto y el día del parto con un BCAD de $9.6 \mathrm{mEq} / 100 \mathrm{~g}$ de MS, y un grupo no suplementado. Se determinó el $\mathrm{pH}$ urinario dos veces por semana entre el día -21 y día 1 del parto. Se determinaron las concentraciones de calcio, fósforo y magnesio sérico los días -21 , $-14,1,15$ y 30 del parto. Se determinó hipocalcemia subclínica si los valores de calcio sérico eran menores de $7.5 \mathrm{mg} / \mathrm{dl}$ los días 1 y 15 posparto. La producción de leche se determinó mediante registros de pesaje mensual. Se encontró una disminución del $\mathrm{pH}$ urinario los días $-14,-7$ y 1 en el grupo suplementado $(\mathrm{p}<0.01)$. La suplementación con $100 \mathrm{~g} / \mathrm{vaca} /$ día de cloruro de calcio en el preparto aumentó las concentraciones séricas de magnesio y fósforo, pero los niveles de calcio sérico se mantuvieron similares a los del grupo no suplementado. La suplementación con cloruro de calcio en el preparto no mejoró la homeostasis del $\mathrm{Ca}^{2+}$ ni evitó la ocurrencia de enfermedades metabólicas como la hipocalcemia y la retención de membranas fetales en el postparto, ni logró incrementar la producción de leche.

Palabras clave: cloruro de calcio; vacas; hipocalcemia; producción de leche

\footnotetext{
${ }^{1}$ Unidad de Investigación en Ciencias Funcionales «Dr. Haity Moussatché» (UNIHM), Facultad de Ciencias Veterinaria, Universidad Centroccidental «Lisandro Alvarado» (UCLA), Barquisimeto, Venezuela

${ }^{2}$ E-mail: isabelmarquez@ucla.edu.ve
}

Recibido: 11 de abril de 2018

Aceptado para publicación: 18 de octubre de 2018 
The aim of this study was to evaluate the effect of pre-partum supplementation with an anionic diet on serum mineral concentrations during the peripartum, occurrence of subclinical hypocalcaemia, retention of foetal membranes and milk production in the first 100 days postpartum. A completely randomized design was used, with 24 Carora cows distributed in two groups: one group supplemented with $100 \mathrm{~g}$ of calcium chloride/cow/day between 21 days before the probable date of calving and the day of calving with a BCAD of $9.6 \mathrm{mEq} / 100 \mathrm{~g}$ of MS, and a non-supplemented group. The urinary $\mathrm{pH}$ was determined twice a week between day -21 and day 1 of parturition. Serum calcium, phosphorus and magnesium concentrations were determined on days $-21,-14,1,15$ and 30 of calving. Subclinical hypocalcaemia was determined if the serum calcium values were lower than $7.5 \mathrm{mg} / \mathrm{dl}$ on days 1 and 15 postpartum. Milk production was determined by monthly weighing records. A decrease in urinary $\mathrm{pH}$ was found on days $-14,-7$ and 1 in the supplemented group $(\mathrm{p}<0.01)$. Supplementation with $100 \mathrm{~g} / \mathrm{cow} /$ day of calcium chloride in the pre-partum increased serum concentrations of magnesium and phosphorus, but serum calcium levels remained like those in the non-supplemented group. Calcium chloride supplementation in the pre-partum did not improve $\mathrm{Ca}^{2+}$ homeostasis or prevent the occurrence of metabolic diseases such as hypocalcaemia and retention of foetal membranes in the postpartum, nor did it increase milk production.

Key words: calcium chloride; cows; hypocalcaemia; milk production

\section{INTRODUCCIÓN}

La cantidad de energía requerida a principios de la lactancia para el mantenimiento de los tejidos corporales y la producción de leche excede la cantidad de energía que la vaca puede obtener de la dieta, ocasionando que la vaca entre en un balance energético negativo (BEN) y comience a utilizar sus reservas de grasa corporal como fuente de energía, lo que desencadena las denominadas enfermedades metabólicas, las cuales son definidas como desórdenes nutricionales en su origen (Goff y Horst, 1997).

Entre los desórdenes metabólicos se encuentran la cetosis, hígado graso, acidosis ruminal (Mulligan y Doherty, 2008), y su mayor incidencia coincide con el periodo próximo al parto y se extiende hasta el pico de la lactancia(Crespi et al., 2006). Aunado a esto, durante las primeras semanas posparto hay una depresión de la función del sistema inmune (Kehrli et al., 1990).
La manipulación del balance catiónanión de la dieta (BCAD) es una práctica común que se emplea en las explotaciones lecheras, la cual consiste en la adición de una sal aniónica a la ración de las vacas secas en el preparto (Block, 1994). La alimentación de las vacas secas con menos contenido de iones $\mathrm{Na}^{+}$y $\mathrm{K}^{+}$con relación al $\mathrm{Cl}^{-}$y el $\mathrm{S}^{-}$, aumenta el $\mathrm{Ca}^{2+}$ disponible en sangre al momento del parto, debido al aumento de la movilización ósea y la absorción intestinal del calcio en respuesta a los cambios en el estado ácido-base del animal. El BCAD se mide en miliequivalentes por cada $100 \mathrm{~g}$ de materia seca (mEq/100g MS) (Lean et al., 2006).

Goff et al. (1991) y Charbonneau et al. (2006) han demostrado que al disminuir el BCAD antes del parto; es decir, al incluir más aniones en la dieta, se reduce el riesgo de hipocalcemia y sus patologías asociadas. Este efecto se debe a que las sales aniónicas inducen una acidificación digestiva y metabólica que favorece la circulación del $\mathrm{Ca}^{2+}$ en el organismo. En líneas generales, con el ma- 
nejo del BCAD lo que se busca es mejorar el balance ácido-base y prevenir los desequilibrios de las vacas al inicio de la lactancia, los cuales están asociados a la presentación de enfermedades metabólicas.

Está demostrado en vacas Holstein que la suplementación con una dieta aniónica durante el preparto puede reducir el riesgo de hipocalcemia clínica y subclínica al inducir una leve acidosis metabólica que contribuye al mejoramiento del equilibrio ácido-base en el animal (Block, 1984; Van Dijk y Lourens, 2001; Mellau et al., 2004; Hu y Kung, 2009). Igualmente, se ha reportado que ayuda a prevenir la retención de membranas fetales (RMF) y mejorar el consumo de MS en el periodo posparto (Wilde, 2006). En este sentido, el presente trabajo tuvo como objetivo generar información sobre el efecto de la suplementación con sales aniónicas en vacas de la raza Carora durante el periparto sobre la concentración sérica de minerales y aspectos productivos.

\section{Materiales y Métodos}

\section{Lugar del Estudio}

El estudio se llevó a cabo bajo una investigación experimental de campo, donde se empleó un diseño longitudinal, correlacional causal. La población objeto de estudio pertenece a una finca comercial de 80 vacas en ordeño ubicada en la parroquia Montañas Verdes del Municipio Torres, estado Lara, Venezuela, cuyo sistema de manejo es semiintensivo. La unidad de producción se encuentra a una altura de $565 \mathrm{msnm}$, ubicada en los valles de la depresión de Carora donde nace la cordillera andina, con una temperatura promedio anual de $24{ }^{\circ} \mathrm{C}$ y una pluviometría de 999 mm (Benítez, 2012).

\section{Animales \\ El estudio fue conducido de acuerdo con el Código de Bioética y Bioseguridad de-}

Cuadro 1. Ingredientes de la ración total mezclada (RTM) con cloruro de calcio ofrecida en el preparto al grupo de vacas suplementado, en base fresca (\%) y $\mathrm{kg} / \mathrm{día}$ de materia fresca (MF)

\begin{tabular}{lcc}
\hline Ingrediente & $\begin{array}{c}\text { Base } \\
\text { fresca } \\
(\%)\end{array}$ & $\begin{array}{c}\mathrm{kg} / \text { día } \\
\mathrm{MF}\end{array}$ \\
\hline Pulpa cítrica húmeda & 26.0 & 5.0 \\
Pasto media calidad & 26.0 & 5.0 \\
Silaje de maíz & 26.0 & 5.0 \\
Alimento preparto & 20.8 & 4.0 \\
Mezcal mineral & 0.5 & 0.1 \\
Cloruro de calcio & 0.5 & 0.1 \\
Procreatin 7 & 0.05 & 0.01 \\
\hline
\end{tabular}

FONACIT-Venezuela (FONACIT, 2009). Para la selección de los animales del estudio se consideraron vacas de 2 o más partos con similares promedios de producción y que se encontrasen entre 245 y 255 días de gestación. La muestra estuvo representada por 24 vacas Carora (Bos taurus), seleccionadas al azar dentro del grupo que cumplía con los criterios de selección, según la fórmula citada por Aguilar-Barojas (2005). De estas, 12 fueron sometidas a una dieta preparto con la adición de $100 \mathrm{~g} / \mathrm{vaca}$ /día de cloruro de calcio (Cuadro 1), que comenzó a administrarse 21 días antes de la fecha probable de parto. Las 12 vacas restantes formaron el grupo control no suplementado. La dieta no fue manipulada en el posparto y ambos grupos recibieron la misma ración (Cuadro 2). Las vacas seleccionadas siguieron el manejo rutinario de la finca.

\section{pH Urinario}

Las muestras se tomaron dos veces por semana desde el día 21 antes de la fecha probable de parto hasta el día del parto y se calculó la media semanal. Fueron colectadas a partir de las 08:30, antes de recibir la ración, 
Cuadro 2. Ingredientes y composición nutricional de la ración total mezclada (RTM) ofrecida en el posparto, en base fresca (\%) y kg/día de materia fresca (MF)

\begin{tabular}{lcc}
\hline Ingrediente & $\begin{array}{c}\text { Base } \\
\text { fresca (\%) }\end{array}$ & $\begin{array}{c}\mathrm{kg} / \text { día } \\
\mathrm{MF}\end{array}$ \\
\hline Silaje de maíz & 40.8 & 15.0 \\
Pulpa cítrica & 21.7 & 8.0 \\
húmeda & 16.3 & 6.0 \\
Gran vaca lechera & 13.6 & 5.0 \\
Afrecho húmedo & 4.0 & 1.5 \\
Heno de bermuda & 2.0 & 0.7 \\
Melaza & 0.5 & 0.2 \\
Energras & 0.2 & 0.1 \\
Clortetraciclina & 0.2 & 0.1 \\
Mineral 14 & 0.2 & 0.06 \\
Azufre & 0.03 & 0.01 \\
Optigen & 0.03 & 0.01 \\
Procreatin 7 & & \\
\hline
\end{tabular}

mediante estimulación de la vulva o masaje en el área perineal. El pH de las muestras $(20 \mathrm{ml})$ de orina colectadas en un recipiente plástico fue determinado de inmediato mediante el uso de un pHmetro (resolución: 0.01; precisión: \pm 0.02 ) (Jorgensen Laboratories, EEUU). El pH urinario permitió monitorear la efectividad de la sal aniónica para acidificar la orina en las vacas que estaban consumiendo la ración total mezclada (RTM) con cloruro de calcio.

\section{Minerales}

En los días 21 y 14 antes de la fecha probable de parto y en los días 1,15 y 30 postparto se extrajeron muestras de sangre mediante punción de la vena coccígea en tubos Vacutainer ${ }^{\circledR}$ sin anticoagulante, y fueron centrifugadas a $3000 \mathrm{x}$ g durante $15 \mathrm{mi}$ nutos. El suero fue trasvasado a tubos Eppendorf® y congelados hasta su análisis. Las muestras para la determinación de calcio, magnesio y fósforo fueron analizadas en la Unidad de Investigación en Ciencias Funcionales «Dr. Haity Moussatché» (UNIHM) del Decanato de Ciencias Veterinarias de la
Universidad Centroccidental «Lisandro Alvarado» (UCLA).

La concentración de los minerales se determinó en un analizador bioquímico veterinario EMP-168Vet (Shenzhen Emperor Electronic Technology, China), utilizando los estuches de Diagnóstico Gamma ${ }^{\circledR}$ para análisis cuantitativo in vitro en suero o plasma de Laboratorios Heiga (Caracas, Venezuela), por medio de técnicas colorimétricas enzimáticas directas, siguiendo las metodologías referida por Michaylova e Illkova (1971) cuyos rangos van entre $2.50 \mathrm{y}$ $15.0 \mathrm{mg} / \mathrm{dl}$ para el calcio, la metodología de Mann y Yoe (1956) para el magnesio con rangos mínimos y máximos de 0.07 y $6.0 \mathrm{mg} / \mathrm{dl}$, respectivamente, y para el fósforo de Daly y Ertingshausen (1972) con rangos de $3.50 \mathrm{a}$ $16.0 \mathrm{mg} / \mathrm{dl}$.

\section{Hipocalcemia Subclínica}

Se determinó los días 1 y 15 posparto y fue definida por valores de calcio sérico por debajo de $7.5 \mathrm{mg} / \mathrm{dl}$ (Goff, 1999).

\section{Producción de Leche}

Se determinó a través de los registros de pesaje mensual de leche en que se realizan en la finca los primeros cinco días de cada mes (am y pm). Se registró la producción de los primeros 100 días de la campaña láctea.

\section{Análisis Estadístico}

El pH urinario, la concentración de los minerales y la producción de leche se analizaron estadísticamente utilizando el siguiente modelo: eYijk $=i+\mathrm{T}_{\mathrm{i}}+\mathrm{C}_{\mathrm{ji}}+\mathrm{D}_{\mathrm{k}}+\mathrm{TD}_{\mathrm{ik}}+$ $\mathrm{e}_{\mathrm{ijk}}$, con el procedimiento PROC MIXED en SAS (2001), donde $Y=$ concentración de los minerales y producción láctea (variable dependiente), $\grave{i}=$ promedio general, $\mathrm{T}_{\mathrm{i}}=$ efecto tratamiento, $\mathrm{C}_{\mathrm{ji}}=$ efecto aleatorio de la vaca dentro del tratamiento, $\mathrm{D}_{\mathrm{k}}=$ efecto fijo del día de muestreo, $\mathrm{TD}_{\mathrm{ik}}=$ interacción de $\mathrm{T} \mathrm{x}$ $\mathrm{D}, \mathrm{e}_{\mathrm{ijk}}=$ error residual. La estructura de covarianza utilizada para los minerales y pro- 


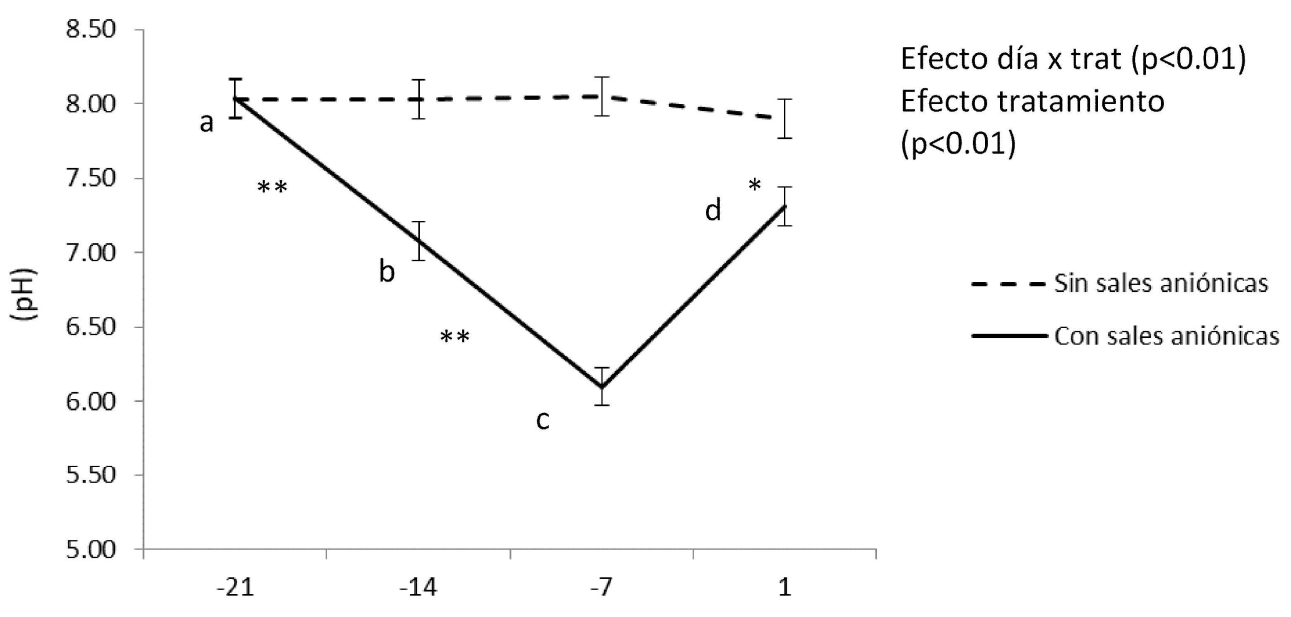

Figura 1. $\mathrm{pH}$ urinario durante el periparto de vacas Carora suplementadas con cloruro de calcio en el preparto. Los valores representan la media \pm error estándar. $n=12$ vacas por grupo. $a, b, c=$ interacción tratamiento $x$ día $(p<0.01)$. a,d $=$ interacción tratamiento $x$ día $(p<0.05)$. *: $p<0.05$; **: $p<0.01$

ducción de leche fue Spatial Power y para el $\mathrm{pH}$ urinario fue Autoregresiva. La comparación de las medias se realizó mediante la prueba de Tukey-Kramer.

Para la determinación de la ocurrencia de casos de hipocalcemia subclínica se aplicó la prueba de los rangos con signo de Wilcoxon con el programa SPSS 15.0 (SPSS, EEUU). Para la retención de membranas fetales se realizó una tabla de contingencia aplicándose una prueba de chi-cuadrado. Para todos los casos se utilizó un nivel de significancia del $95 \%$.

\section{Resultados y Discusión}

\section{pH Urinario}

El control del pH de la orina es una forma efectiva de determinar si los animales están consumiendo niveles adecuados de productos aniónicos en relación con los niveles de potasio en la ración (Espino et al., 2005). Los valores de $\mathrm{pH}$ en orina reportados para vacas Holstein están comprendidos entre 6.2 y 6.0 , mientras que un $\mathrm{pH}$ entre 5.5 y 6.0 podrían ser necesarios en vacas Jersey para una respuesta óptima (Hutjens, 2013). Si bien hay posibles diferencias atribuibles a la raza, no se disponen de valores de referencia de este parámetro para vacas Carora.

Los valores de $\mathrm{pH}$ urinario en el grupo no suplementado del presente estudio se mantuvieron constantes durante todo el ensayo en comparación con los valores del grupo suplementado, que fueron disminuyendo progresivamente a partir del día -14 (7.08 \pm 0.13$)$, alcanzando sus niveles más bajos el día -7 (6.11 \pm 0.13$)$, encontrándose diferencias altamente significativas $(p<0.01)$ entre tratamientos para los días $-14,-7$ y 1 . Como era de esperarse, el $\mathrm{pH}$ aumentó inmediatamente después del parto en el grupo suplementado (Figura 1).

Estos resultados coinciden con lo reportado por Goff y Horst (2003), quienes indican que el pH urinario se encuentra alrededor de 8.2 para dietas catiónicas y entre 5.8 y 6.2 para dietas aniónicas. Según Horst et al. (1997), pueden aparecer signos clínicos 
de acidosis metabólica con valores de $\mathrm{pH}$ por debajo de 5.5; no obstante, Espino et al. (2005) afirman que los mecanismos homeostáticos son capaces de amortiguar la acidosis metabólica y mantener el $\mathrm{pH}$ sanguíneo dentro del rango fisiológico, ante una gran cantidad de sales aniónicas.

Los resultados obtenidos también concuerdan con Block (1994), quién argumenta que este tipo de dieta puede modificar el balance aniónico-catiónico y provocar la reducción del pH urinario (Calsamiglia et al., 2012). Por su parte, Goff (2008) ha demostrado que la diferencia entre el número de partículas absorbidas de cationes y aniones determina el balance ácido-base del organismo y, por lo tanto, el pH de la sangre, el cual está altamente relacionado al $\mathrm{pH}$ urinario. Recientemente, Guzmán et al. (2016) trabajando con vacas Holstein encontraron una correlación positiva de 0.331 entre los valores del balance catión anión en la dieta (DCAD) y el pH urinario.

No se dispone de trabajos científicos en la raza Carora donde se establezca el valor de $\mathrm{pH}$ urinario. En la presente investigación, a pesar de que hubo vacas cuyos valores se encontraron por debajo de 5.5, estas no manifestaron signo clínico alguno de acidosis, lo que permite inferir que el $\mathrm{pH}$ urinario para las vacas de esta raza, suplementadas con cloruro de calcio, se encuentran por debajo de los valores reportados para otras razas, sin causar efectos negativos en el animal.

\section{Concentración Sérica de Minerales}

El calcio $\left(\mathrm{Ca}^{2+}\right)$ mantiene la integridad de la estructura de huesos y dientes y es fundamental para controlar diversos procesos bioquímicos. E1 99\% del calcio se encuentra en los huesos, $1 \%$ en el citosol de las células y un $0.3 \%$ en el líquido extracelular (Horst et al., 1994). Por otro lado, el 55\% del calcio plasmático total se encuentra en forma ionizada, como $\mathrm{Ca}^{2+}$ activo, el $35 \%$ está unido a proteínas, principalmente albúmina y el
$10 \%$ constituye complejos en formas no iónicas como el bicarbonato de calcio (Albornoz et al., 2016). El equilibrio entre el $\mathrm{Ca}^{2+}$ ionizado y el unido a proteínas depende del pH sanguíneo. La alcalosis aumenta este último y disminuye la concentración de $\mathrm{Ca}^{2+}$ mientras que la acidosis tiene el efecto opuesto (Albornoz et al., 2016). Los niveles de $\mathrm{Ca}^{2+}$ sanguíneo en vacas lecheras sin suplementación con sales aniónicas varían entre 8.60 y $9.69 \mathrm{mg} / \mathrm{dl}$ (Goff, 2008).

En el presente estudio, las concentraciones de $\mathrm{Ca}^{2+}$ sérico permanecieron ligeramente superiores en los días 1 y 15 posparto $(7.44 \pm 0.51$ y $8.60 \pm 0.51 \mathrm{mg} / \mathrm{dl})$ en el grupo suplementado con respecto al grupo no suplementado $(6.74 \pm 0.51$ y $7.17 \pm 0.51 \mathrm{mg} / \mathrm{dl})$, aunque sin diferencias significativas por efecto del tratamiento. No obstante, hubo interacción entre los días muestreados (Figura 2). En el día 30 posparto la media para el grupo suplementado fue de $10.54 \pm 0.51 \mathrm{mg} / \mathrm{dl}$ vs. $8.92 \pm 0.51 \mathrm{mg} / \mathrm{dl}$ en el grupo no suplementado, lo cual podría deberse a que el efecto del cloruro de calcio (administrado en el preparto) sobre los niveles de calcio iónico se manifiesta mayormente en las primeras semanas posparto. Como era esperado, la movilización de este mineral comenzó desde los días previos al parto.

Hu et al. (2007) tampoco encontraron diferencias significativas en vacas Holstein en las concentraciones séricas de $\mathrm{Ca}^{2+}$ en el posparto utilizando un BCAD de $-3 \mathrm{mEq} / 100 \mathrm{~g}$ de MS, con $0.85 \%$ de cloruro de calcio en la RTM, al igual que DeGroot et al. (2010) con un BCAD de - $10 \mathrm{mEq} / 100 \mathrm{~g}$ de MS. Así mismo, Seifi et al. (2010) han señalado un aumento significativo al utilizar un BCAD de $82 \mathrm{mEq} / 100 \mathrm{~g}$ de MS con respecto al grupo control. Por su parte, Ramos-Nieves et al. (2009) observaron que las concentraciones cálcicas se incrementaron significativamente durante las primeras 24 horas posparto en el grupo suplementado con un BCAD de -15 $\mathrm{mEq} / 100 \mathrm{~g}$ de $\mathrm{MS}$ (6.3\% de SoyChlor $\left.{ }^{\circledR}\right)$ en el periodo preparto. 


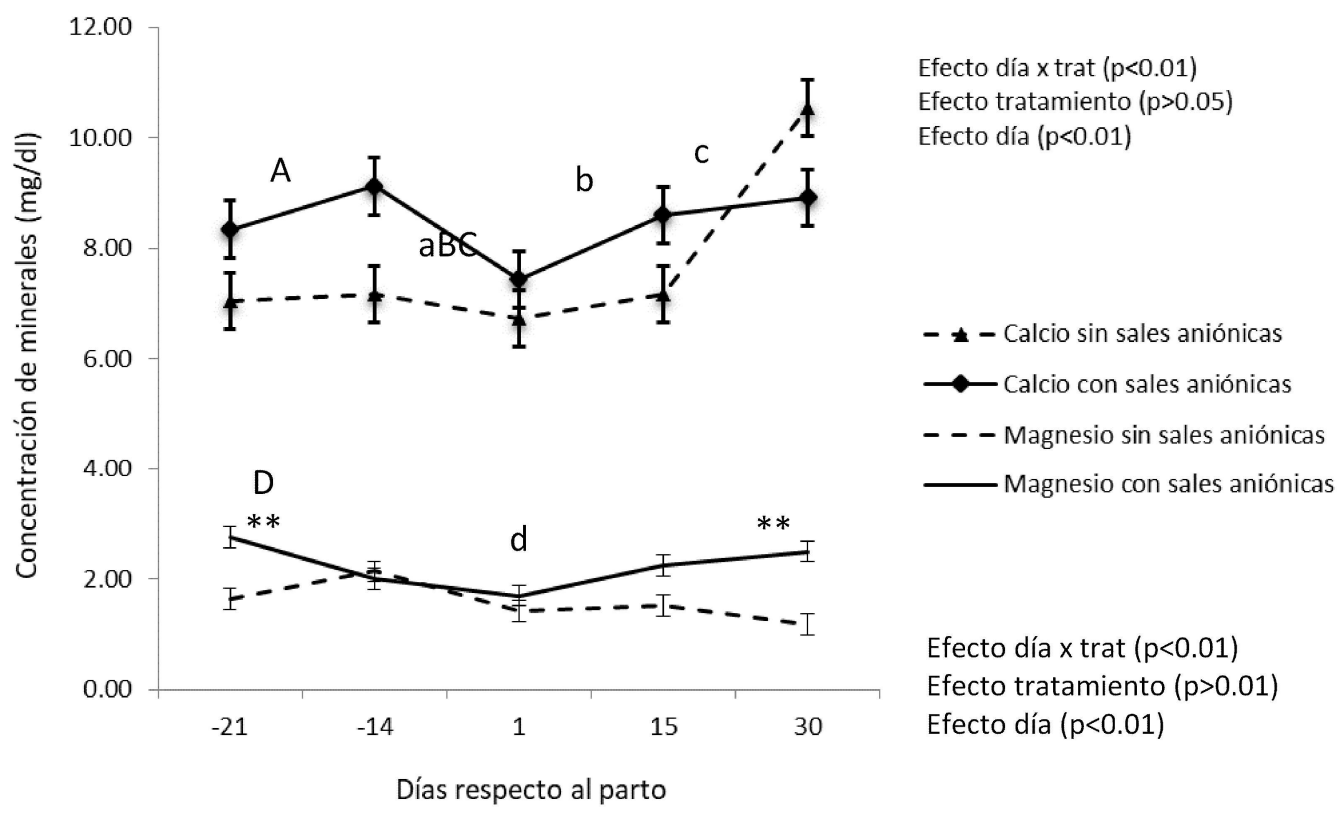

Figura 2. Concentraciones séricas de calcio y magnesio durante el periparto de vacas Carora suplementadas con cloruro de calcio en el preparto. Los valores representan la media \pm error estándar. $n=12$ vacas por grupo. Aa = interacción tratamiento $x$ día $(p<0.01) ; B b=$ interacción tratamiento $x$ día $(p=0.05) ; C c=$ interacción tratamiento $x$ día $(p<0.05)$; ef = interacción tratamiento $x$ día $(p<0.01)$; **: $p<0.01$

Las razones que expliquen la ausencia de efecto de la sal aniónica posiblemente estén relacionadas al bajo nivel de magnesio encontrados al día posterior al parto en vacas suplementadas. Las concentraciones séricas de magnesio en vacas del grupo suplementado mostraron diferencias altamente significativas $(\mathrm{p}<0.01)$ al día -21 del preparto y 30 posparto entre el grupo suplementado $(2.76$ y $2.50 \pm 0.19 \mathrm{mg} / \mathrm{dl})$ y el no suplementado 1.65 y $1.18 \pm 0.18 \mathrm{mg} / \mathrm{dl})$. En el grupo suplementado con cloruro de calcio se evidenció un aumento desde el día $1(1.70 \pm 0.18$ $\mathrm{mg} / \mathrm{dl}$ ) hasta el día 30 posparto, mientras que en el no suplementado se registró un descenso a partir del día $15(1.52 \pm 0.19 \mathrm{mg} / \mathrm{dl})$, con las concentraciones más bajas en el día 30 posparto (Figura 2). Goff (2008) sugiere que con concentraciones séricas de magnesio por debajo de $1.5 \mathrm{mg} / \mathrm{dl}$ en el periodo posparto se puede incrementar la susceptibilidad de la vaca a presentar hipocalcemia.

En el presente estudio, ambos grupos registraron vacas con concentraciones séricas de magnesio por debajo de $1.5 \mathrm{mg} / \mathrm{dl}$ en el día 1 posparto. DeGaris y Lean (2008) afirman que la hipomagnesemia posparto es la segunda causa de hipocalcemia, debido a que el magnesio es un cofactor necesario para estimular la producción de AMPc por parte de la paratohormona (PTH). En efecto, las bajas concentraciones de magnesio traen como consecuencia la incapacidad del hueso y del riñón para responder ante la PTH para regular el $\mathrm{Ca}^{2+}$ sérico. Estos resultados difieren de los reportados por Ramos-Nieves et al. (2009), quienes utilizando $6.3 \%$ de 


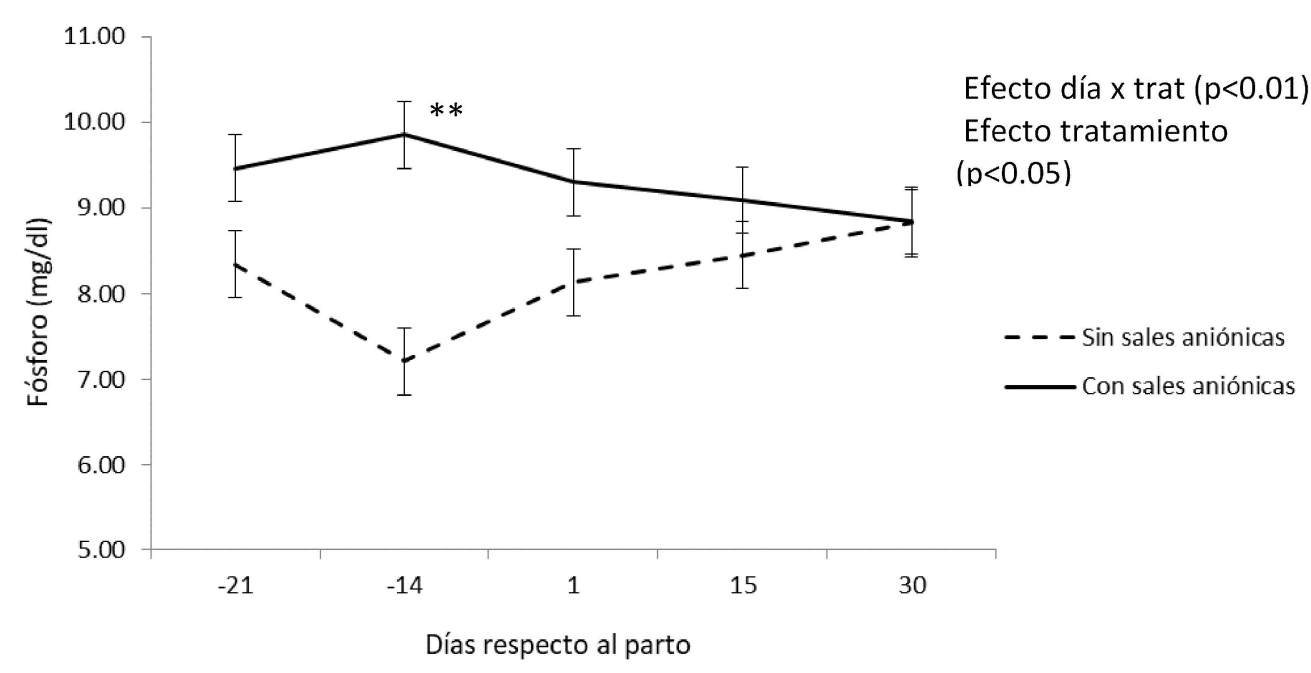

Figura 3. Concentraciones séricas de fósforo durante el periparto de vacas Carora suplementadas con cloruro de calcio en el preparto. Los valores representan la media \pm error estándar. $n=12$ vacas por grupo. **: $p<0.05$

SoyChlor ${ }^{\circledR}$ del total de la RTM para lograr un BCAD de $-15 \mathrm{mEq} / 100 \mathrm{~g}$ de MS, no encontraron diferencias significativas en las concentraciones de magnesio sérico ni en la incidencia de hipomagnesemia al parto entre los grupos sometidos a una dieta aniónica preparto y el grupo control.

Las concentraciones séricas de fósforo presentaron diferencias altamente significativas $(p<0.01)$ al día -14 entre grupos $(9.85 \pm 0.39$ para el grupo suplementado vs. $7.21 \pm 1.61 \mathrm{mg} / \mathrm{dl}$ para el grupo control). Las demás determinaciones de fósforo fueron similares entre grupos (Figura 3). Estos resultados contrastan con los reportado por Ramos-Nieves et al. (2009) donde los valores de fósforo sérico se incrementaron en el primer día posparto al suministrar una dieta con un BCAD de $-15 \mathrm{mEq} / 100 \mathrm{~g}$ de MS 21 días antes del parto. Por otro lado, Chan et al. (2006) y León et al. (2008) no encontraron diferencias significativas en fósforo sérico al suministrar 0.99 y $1.50 \%$ de cloruro de calcio en la RTM para obtener un BCAD de -6 y $-48 \mathrm{mEq} / 100 \mathrm{~g}$ de $\mathrm{MS}$, respectivamente.

\section{Presentación de Hipocalcemia}

La reducción del pH urinario no se tradujo en la prevención de hipocalcemia en las vacas suplementadas con $100 \mathrm{~g}$ de cloruro de calcio/vaca/día. En ambos grupos se registró el 50\% de vacas con concentraciones de $\mathrm{Ca}^{2+}$ sérico al parto entre 5.5 y $7.5 \mathrm{mg} / \mathrm{dl}$, mientras que el $33.3 \%$ de las vacas del grupo suplementado y el $41.7 \%$ del grupo no suplementado en el día 15 posparto estuvieron afectadas (Figura 4).

Según Goff (2008), para un óptimo control y reducción del riesgo de hipocalcemia, el promedio del $\mathrm{pH}$ urinario debería estar entre 5.8 y 6.2 en vacas Holstein. Los resultados de esta investigación contrastan con los obtenidos con otras razas de vacas europeas. Así, Charbonneau et al. (2006), en un metaanálisis donde se estudiaron 75 grupos de tratamientos en diversas razas lecheras, se confirmó la efectividad de la disminución del BCAD con un $\mathrm{pH}$ urinario de 6.0 para la prevención de hipocalcemia en el posparto. Por su parte, Kurosaki et al. (2007) reporta- 


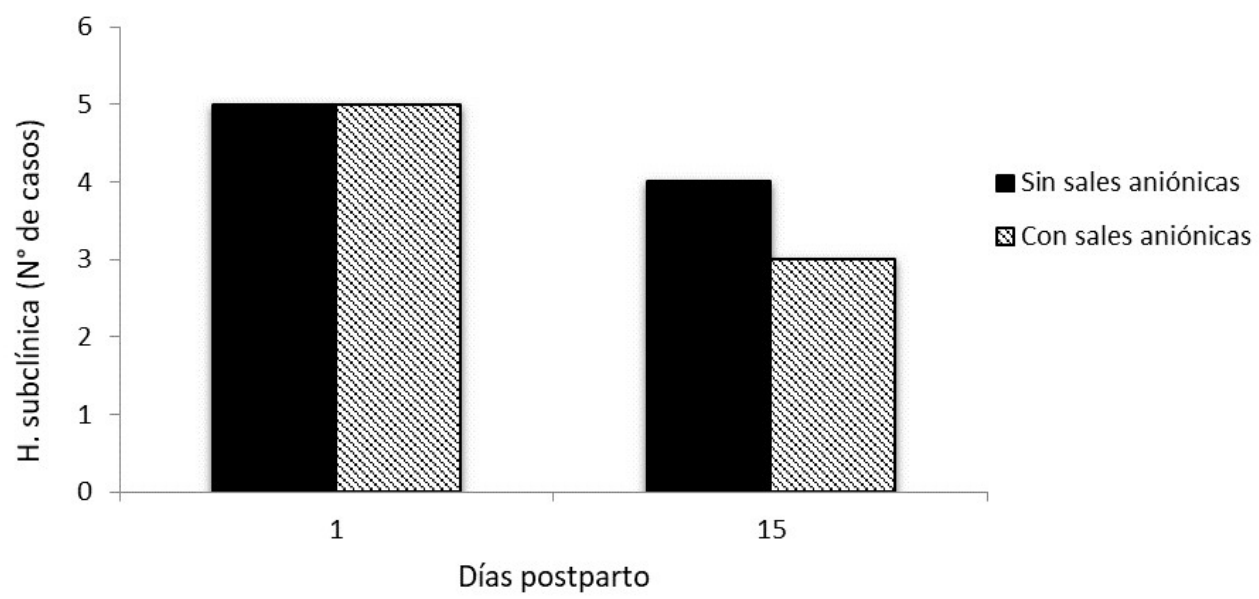

Figura 4. Número de casos de hipocalcemia subclínica en vacas Carora suplementadas con cloruro de calcio en el preparto

ron una disminución del $50 \%$ en la incidencia de hipocalcemia subclínica con niveles de $\mathrm{pH}$ urinario de 6.8-7.0 en vacas suplementadas con sales aniónicas en comparación con las vacas del grupo control.

En coincidencia con los resultados del presente estudio, Ramos-Nieves et al. (2009) concluyeron que la suplementación con un BCAD de $-15 \mathrm{mEq} / 100 \mathrm{~g}$ de MS no afectó la incidencia de hipocalcemia clínica y subclínica. Dishington (1975), pionero en la utilización de las sales aniónicas para la alimentación de vacas secas, concluyó que estas incidencias presentan grandes variaciones entre rebaños, composición racial y tipo de dieta.

En este estudio se pudo evidenciar que la ligera acidosis metabólica inducida con la adición de sales aniónicas a la ración no contribuyó al mejoramiento de la homeostasis del $\mathrm{Ca}^{2+}$, y no redujo los casos de hipocalcemia subclínica en comparación con el grupo control. Posiblemente, en vacas Carora se requiera utilizar una dosis superior para lograr una mayor acidificación o utilizar otra sal aniónica más eficiente para mejorar los niveles de $\mathrm{Ca}^{2+}$ sérico. Así mismo, considerando que los niveles de magnesio en el día 1 del posparto estuvieron por debajo $2 \mathrm{mg} / \mathrm{dl}$, es probable que una hipomagnesemia en la primera semana posparto haya sido un factor determinante en la hipocalcemia observada.

\section{Retención de Membranas Fetales}

La RMF se define como la falla para expulsar las membranas fetales dentro de las 12-24 horas después del parto. Entre los factores de riesgo para el establecimiento de esta patología se encuentran los abortos, partos distócicos, deficiencias nutricionales y enfermedades metabólicas, principalmente hipocalcemia, entre otros (Meléndez et al., 2004). Debido a que no se ha demostrado la efectividad de los tratamientos más comúnmente utilizados para evitar la RMF, la prevención a través de la manipulación del BCAD en el preparto es una herramienta relevante (Beagley et al., 2010).

En este estudio, la proporción de vacas con RMF fue ligeramente mayor para el grupo suplementado con sales aniónicas $(25.0 \%)$ con respecto al no suplementado (16.7\%), pero sin diferencias significativas. Estos re- 
sultados coinciden con otros autores (Goff y Horst, 1997; Joyce et al., 1997) donde las sales aniónicas no tuvieron efecto sobre la prevención de esta patología.

Meléndez et al. (2004) asociaron la disminución de $\mathrm{Ca}^{2+}$ sérico en las primeras seis horas del parto con la presentación de RMF; sin embargo, Beagley et al. (2010) argumentan que esta patología está más asociada con una disminución del sistema inmune en el animal.

\section{Producción de Leche}

La producción de leche en el grupo suplementado fue de $13.5 \pm 0.9 \mathrm{~L}$ y en el grupo control fue $13.6 \pm 1.0 \mathrm{~L}$, sin diferencias significativas entre grupos. Sin embargo, se observó un aumento significativo $(\mathrm{p}<0.05)$ de la producción en el día 80 en el grupo que consumió sales aniónicas. Hu et al. (2007) tampoco hallaron diferencias significativas en términos de producción de leche entre vacas que consumieron sales aniónicas y vacas control; mientras que Galvis et al. (2007) indican que vacas sometidas a un BCAD negativo llegan a aumentar la producción de leche.

Es posible que al incrementar el número de días preparto con suplementación aniónica se evidencie un efecto positivo en los promedios de producción láctea. DeGroot et al. (2010) reportaron un aumento de la producción de leche en vacas Holstein con un BCAD de $-12 \mathrm{mEq} / 100 \mathrm{~g}$ de MS, mientras que Weich et al. (2013) obtuvieron resultados similares en vacas Holstein sometidas a un BCAD de $-16 \mathrm{mEq} / 100 \mathrm{~g}$ de MS durante 42 días antes del parto.

\section{Conclusiones}

- La suplementación con cloruro de calcio en el preparto no mejoró la homeostasis del $\mathrm{Ca}^{2+}$ ni evitó la ocurrencia de enfermedades metabólicas como la hipocalcemia sérica y la retención de membranas fetales en el postparto, ni logró incrementar la producción de leche.

- La suplementación con 100 g/vaca/día de cloruro de calcio en el preparto aumentó las concentraciones séricas de magnesio y fósforo, pero los niveles de calcio sérico se mantuvieron similares a los del grupo no suplementado.

- La medición del pH urinario proporciona una evaluación sencilla y es un indicador apropiado de la suplementación de aniones en la dieta.

\section{Agradecimientos}

Al Consejo de Desarrollo Científico, Humanístico, Tecnológico (CDCHT) por el registro del Proyecto 006-RCV-2015 y a la Fundación Observatorio Nacional de Ciencia, Tecnología e Innovación (ONCTI) por el financiamiento de este proyecto.

\section{Literatura Citada}

1. Aguilar-Barojas S. 2005. Fórmulas para el cálculo de la muestra en investigaciones de salud. Salud en Tabasco 11: 333-338.

2. Albornoz L, Albornoz J, Morales M, Fidalgo L. 2016. Hipocalcemia puerperal bovina. Veterinaria 52: 28-38.

3. Beagley JC, Whitman KJ, Baptiste KE, Scherzer J. 2010. Physiology and treatment of retained fetal membranes in cattle. J Vet Intern Med 24: 261-268. doi: $10.1111 /$ j.1939-1676.2010.0473.x

4. Benitez O. 2012. Demarcaciones climáticas del municipio Torres en el estado Lara, Venezuela. Agronomía Trop 62: 1-4.

5. Block E. 1984. Manipulating dietary anions and cations for prepartum dairy cows to reduce incidence of milk fever. J Dairy Sci 67: 2939-2948. doi: 10.3168/ jds.S0022-0302(84)81657

6. Block E. 1994. Manipulation of dietary cation-anion difference on nutritionally related production diseases, productivity, 
and metabolic responses of dairy cows. J Dairy Sci 77: 1437-1450. doi: 10.3168/ jds.S0022-0302(94)77082-X

7. Calsamiglia S, Blanch M, Ferret A, Moya D. 2012. ¿Es la acidosis un problema asociado al $\mathrm{pH}$ ? Causas y herramientas para su control. En: XXVIII Curso de Especialización FEDNA. Madrid. [Internet]. Disponible en: http:// fundacionfedna.org/sites/default/files/ 12Cap_VI.pdf

8. Chan PS, West JW, Bernard JK. Effect of prepartum dietary calcium on intake and serum and urinary mineral concentrations of cows. J Dairy Sci 89: 704-713. doi: 10.3168/jds.S00220302(06)72133-6

9. Charbonneau E, Pellerin D, Oetzel GR. 2006. Impact of lowering dietary cation-anion difference in nonlactating dairy cows: a meta-analysis. J Dairy Sci 89: 537-548. doi: 10.3168/jds.S00220302(06)72116-6

10. Crespi D, Medín J, Piana M, Piferrer G, Meikle A, Uriarte G, et al. 2006. Efecto de la suplementación energética y del suministro de sales aniónicas durante el preparto sobre la producción y reproducción en vacas Holstein en pastoreo. Veterinaria 41: 9-20.

11. Daly JA, Ertingshausen G. 1972. Direct method for the determining inorganic phosphate in serum with the «CentriFichem». Clin Chem 18:263-265.

12. Daniel RC. 1983. Motility of the rumen and abomasum during hypocalcaemia. Can J Comp Med 47: 276-280.

13. DeGaris, P; Lean, I. 2008. Milk fever in dairy cows: a review of pathophysiology and control principles. Vet J 176: 58-69. doi: 10.1016/j.tvj1.2007.12.029

14. DeGroot MA, Block E, French PD. 2010. Effect of prepartum anionic supplementation on periparturient feed intake, health, and milk production. $\mathrm{J}$ Dairy Sci 93: 5268-5279. doi: 10.3168/ jds.2010-309

15. Dishington IW. 1975. Prevention of milk fever (hypocalcemic paresis puerperalis) by dietary salt supplements. Acta Vet Scand 16: 503-512.
16. Espino L, Suárez M, Santamarina G, Goicoa A, Fidalgo L. 2005. Utilización de las sales aniónicas en la prevención de la paresia puerperal hipocalcémica. Arch Med Vet 37: 7-13. doi: 10.4067/ S0301-732X2005000100002.

17. [FONACIT] Fondo Nacional de Ciencia, Tecnología e Innovación. 2009. Norma para utilización de animales en investigación. En: Código de bioética y bioseguridad. $3^{\circ}$ ed. Caracas, Venezuela: $\mathrm{p}$ 33-35.

18. Galvis RD, Agudelo D, Saffon A. 2007. Condición corporal, perfil de lipoproteínas y actividad ovárica en vacas Holstein en lactancia temprana. Rev Colomb Cienc Pec 20: 16-29.

19. Goff J. 1999. Treatment of calcium, phosphorus, and magnesium balance disorders. Vet Clin North Am Food Anim Pract 15: 619-639.

20. Goff JP. 2008. The monitoring, prevention, and treatment of milk fever and subclinical hypocalcaemia in dairy cows. Vet J 176: 50-57. doi: 10.1016/ j.tvj1.2007.12.020

21. Goff J, Horst R. 1997. Physiological changes at parturition and their relationship to metabolic disorders. J Dairy Sci 80: 1260-1268. doi: 10.3168/ jds.S0022-0302(97)76055-7

22. Goff J, Horst R. 2003. Milk fever control in the United States. Acta Vet Scand 97: 145-147.

23. Goff JP, Horst RL, Mueller FJ, Miller JK, Kiess GA, Dowlen HH.1991. Addition of chloride to a prepartal diet high in cations increases 1,25-dihydroxyvitamin $\mathrm{D}$ response to hypocalcemia preventing milk fever. J. Dairy Sci 74: 3863 - 3871. doi: 10.3168/jds.S00220302(91)78579-2

24. Guzmán A, Montoya B, Gómez CA. 2016. Determinación del balance catión anión dietario en vacas de preparto en establos lecheros de Lima y Trujillo, Perú, y su relación con el $\mathrm{pH}$ urinario. Rev Inv Vet Peru 27: 698-705. doi: 10.15381/rivep.v27i4.12559 
25. Horst RL; Goff, JP; Reinhardt, TA. 1994. Calcium and vitamin D metabolism in the dairy cow. J Dairy Sci 77: 1936-1951. doi: 10.3168/jds.S00220302(94)77140-X

26. Horst RL, Goff JP, Reinhardt TA, Buxton DR. 1997. Strategies for preventing milk fever in dairy cattle. $\mathrm{J}$ Dairy Sci 80: 1269-1280. doi: 10.3168/ jds.S0022-0302(97)76056-9

27. Hu W, Kung Jr L. 2009. Effect of dietary ratio of $\mathrm{Na}: \mathrm{K}$ on feed intake, milk production, and mineral metabolism in mid-lactation dairy cows. J Dairy Sci 92: 2711-2718. doi: 10.3168/jds.2008-1231

28. Hu W, Murphy MR, Constable PD, Block E. 2007. Dietary cation-anion difference and dietary protein effects on performance and acid-base status of dairy cows in early lactation. J Dairy Sci 90:3355-3366. doi: 10.3168/jds.2006-514

29. Hutjens M. 2013. Fisiología digestiva y uso de aditivos en rumiantes. En: XXIX Curso de Especialización FEDNA. Madrid. [Internet]. Disponible en: http:// fundacionfedna.org/sites/default/files/ 13CAP_IItrad.pdf

30. Joyce PW, Sanchez WK, Goff JP. 1997. Effect of anionic salts in prepartum diets based on alfalfa. J Dairy Sci 80: 2866-2875. doi: $10.3168 /$ jds.S0022-0302(97)76251-9

31. Kehrli ME, Goff JP, Harp JA, Thurston JR, Norcross NL. 1990. Effects of preventing hematology, conglutinin, immunoglobulin, and shedding of Staphylococcus aureus in milk. J Dairy Sci 73: 2103-2111. doi: 10.3168/jds.S0022-0302(90)78890-X

32. Kurosaki N, Yamato O, Sato J, Naito Y, Mori F, Imoto S, Maede Y. 2007. Biomarkers for the activation of calcium metabolism in dairy cows: elevation of tartrate-resistant acid phosphatase activity by lowering dietary cation-anion difference is associated with the prevention of milk fever. J Vet Med Sci 69:265-270.
33. Lean IJ, DeGaris PJ, McNeil DM, Block E. 2006. Hypocalcemia in dairy cows: meta-analysis and dietary cationanion difference theory revisited. J Dairy Sci 89: 669-684. doi: 10.3168/jds.S00220302(06)72130-0

34. León J, Mojica J, Castro E, Cárdenas E, Pabón M, Carulla J. 2008. Balance de nitrógeno y fósforo de vacas lecheras en pastoreo con diferentes ofertas de kikuyo (Pennisetum clandestinum) suplementadas con ensilaje de avena (Avena sativa). Rev Colomb Cienc Pec 21: 559-570.

35. Mann C, Yoe J. 1956. Spectrophotometric determination of magnesium with sodium 1-Azo-2-hydroxy-3-(2,4dimethylcarboxanilido)-naphthalene-1'(2-hydroxybenzene-5-sulfonate). Anal Chem 28: 202-205. doi: 10.1021/ ac60110a016

36. Meléndez P, Donovan GA, Risco CA, Goff JP. 2004. Plasma mineral and energy metabolite concentrations in dairy cows fed an anionic prepartum diet that did or did not have retained fetal membranes after parturition. Am J Vet Res 65: 1071-1076. doi: 10.2460/ ajvr.2004.65.1071

37. Mellau LS, Jorgensen RJ, Bartlett PC, Enemark JM, Hansen AK. 2004. Effect of anionic salt and highly fermentable carbohydrate supplementations on urine $\mathrm{pH}$ and on experimentally induced hypocalcemia in cows. Acta Vet Scand 45: 139-147. doi: 10.1186/17510147-45-139

38. Michaylova V, Illkova P. 1971. Photometric determination of micro amounts of calcium with Arsenazo III. Anal Chim Acta 53: 194-198. doi: 10.1016/S0003-2670(01)80088-X

39. Mulligan F, Doherty M. 2008. Production diseases of the transition cow. Vet J 176: 3-9. doi: 10.1016/ j.tvj1.2007.12.018

40. Ramos-Nieves JM, Thering BJ, Waldron MR, Jardon PW, Overton TR. 2009. Effects of anion supplementation to low-potassium prepartum 
diets on macromineral status and performance of periparturient dairy cows. J Dairy Sci 92: 5677-5691. doi: 10.3168/ jds.2009-2378

41. Seifi HA, Mohri M, Kalamati-Zadeh J. 2004. Use of pre-partum urine $\mathrm{pH}$ to predict the risk of milk fever in dairy cows. Vet J 167: 281-285. doi: 10.1016/ S1090-0233(03)00114-X

42. Van Dijk CJ, Lourens DC. 2001. Effects of anionic salts in a prepartum dairy ration on calcium metabolism. J S Afr Vet Assoc 72: 76-80.
43. Weich W, Block E, Litherland NB.. 2013. Extended negative dietary cationanion difference feeding does not negatively affect postpartum performance of multiparous dairy cows. J Dairy Sci 96: 5780-5792. doi: 10.3168/jds.20126479

44. Wilde D. 2006. Influence of macro and micro minerals in the peri-parturient period on fertility in dairy cattle. Anim Reprod Sci 96: 240-249. doi: 10.1016/ j.anireprosci.2006.08.004 